\title{
Rofecoxib, in contrast to celecoxib, does not have direct antimicrobial effect and does not cause small intestinal dysbiosis in the rat
}

\section{Zoltan S. Zadori ${ }^{1}$, Bernadette Lazar ${ }^{1}$, Gabor Brenner ${ }^{1}$, Szilvia Laszlo ${ }^{1}$, Emese Bato ${ }^{2}$, Eszter Ostorhazi ${ }^{3}$, Janos Juhasz ${ }^{4}$, Dora Szabo ${ }^{3}$, Peter Ferdinandy ${ }^{1}$, Klara Gyires ${ }^{1}$}

\author{
${ }^{I}$ Department of Pharmacology and Pharmacotherapy, Semmelweis University, Hungary, ${ }^{2}$ Second Department of \\ Internal Medicine and Cardiology Center, University of Szeged, Hungary, ${ }^{3}$ Institute of Medical Microbiology, \\ Semmelweis University, Hungary, ${ }^{4}$ Faculty of Information Technology and Bionics, Pazmany Peter Catholic University, \\ Hungary
}

Background: Nonsteroidal anti-inflammatory drugs were shown to induce small intestinal dysbiosis by causing a shift from Gram-positive to predominantly Gram-negative bacteria. This dysbiosis may contribute significantly to the development of small intestinal mucosal injury. It is, however, still not clear whether selective cyclooxygenase-2 (COX-2) inhibitors that are much safer for the gastrointestinal tract, have any effect on the composition of small intestinal microflora. Although celecoxib was shown to induce dysbiosis in mice (and may have such effect in humans as well), it also has direct antibacterial effect against Gram-positive strains, therefore its dysbiotic effect may be unrelated to COX-2 inhibition. Thus, in the present study we aimed to evaluate the effects of another selective COX-2 inhibitor, rofecoxib, 1) on the small intestinal microbiota in the rat, and 2) on the growth of distinct Gram-positive and Gram-negative bacteria in vitro. Methods: Male Wistar rats were treated with either rofecoxib $(5.12 \mathrm{mg} / \mathrm{kg}$ once daily) or with its vehicle (1\% methylcellulose) for 4 weeks, then the jejunal luminal content was collected, and the composition of its microflora was determined by deep sequencing of $16 \mathrm{~S}$ rRNA. The direct effect of rofecoxib on the growth of various bacteria was examined by the broth microdilution assay. Results: 1. Celecoxib inhibited the growth of various Gram-positive bacteria, with MICs ranging from 32-64 mg/l, whereas Gram-negative bacteria were not affected. In contrast, rofecoxib (up to 256 $\mathrm{mg} / \mathrm{l})$ had no significant inhibitory effect on the growth of any of the bacteria tested. 2. The 4-week treatment with rofecoxib did not influence significantly the abundance of the identified small intestinal organisms and had no significant effect on the diversity of microbiota. Conclusions: This study demonstrates that selective COX-2 inhibition by rofecoxib does not influence the small intestinal microbiota, and suggests that the observed dysbiotic effect of celecoxib is due to its direct antibacterial property. However, this should be confirmed by further studies using other selective COX-2 inhibitors. The research was supported by the European Foundation for Disease Research (EFSD) New Horizons Collaborative Research Initiative and National Research, Development and Innovation Office of Hungary (NKFI FK 124878; NVKP-16 -1-2016-0017 National Heart Program). 